Rev. High Pressure Sci. Technol., Vol. 7 (1998) 263 265

\title{
High Pressure Studies of the FCC Chromium Hydride
}

\author{
M. Tkacz \\ Institute of Physical Chemistry, Polish Academy of Sciences, \\ Kasprzaka 44/52, 01-224 Warsaw, Poland
}

\begin{abstract}
The equilibrium pressure of hydrogen gas above solid face centered cubic (fcc) phase of chromium hydride has been determined at $150^{\circ} \mathrm{C}$. As compared to the hexagonal close packed (hcp), chromium hydride plateau for the $\alpha+\beta$ phase region has been found to be lower by about 500 bars and equal to 0.25 $\pm 0.01 \mathrm{GPa}$. The search for possible pressure induced phase transition from the fcc to the hcp structure has been unsuccessful up to $37 \mathrm{GPa}$. The compressibility of the fcc hydride is similar to that of the hcp phase, showing pressure independence of the hydrogen volume in the metal lattice, and confirming the general rule observed in transition metal hydrides.
\end{abstract}

[high pressure, chromium hydride, thermodynamics, equation of state]

\section{Introduction}

Depending on conditions of electrodeposition, chromium can form two different hydrides[1-5], both highly saturated with hydrogen. The hexagonal hydride was the object of systematic thermodynamic investigations by different authors[6-12], while the second of fcc nature is hardly explored. The reason for the lack of information about the fcc hydride is due to difficulties in preparation of this hydride in a reproducible way. Estimation of the equilibrium conditions, which eventually led to successful synthesis came from the pioneering work measuring equilibrium pressure of the hydrogen gas above chromium hydride at $150^{\circ} \mathrm{C}$ by Baranowski and Bojarski [8]. The value of hydrogen equilibrium pressure of about $0.3 \mathrm{GPa}$ was used to calculate the standard thermodynamic potential of the chromium hydride formation as equal to: 4128 $\pm 80 \mathrm{cal} / \mathrm{mole} \mathrm{H}_{2}$. Temperature versus pressure phase diagram for the hcp chromium hydride has been evaluated by Ponyatovsky et al.[12] on the basis of resistometric experiments, showing the typical for the metal-hydride systems hysteresis, which persists at temperatures up to $400^{\circ} \mathrm{C}$.

The main purposes of this paper are: firstly to establish the thermodynamic characteristics of the fcc chromium hydride by the equilibrium hydrogen pressure above the solid hydride phase, secondly, searching pressure induced phase transition and third to determine the equation of state in the pressure range up to $37 \mathrm{GPa}$ at ambient temperature.

\section{Experimental}

a.) Sample preparation and characterization: We have found, that the temperature of electrodeposition is the crucial factor in the successful synthesis of fcc chromium hydride. Basically, we have adopted the method described by Stock and Hardcastle[4] with a few modifications. Firstly, we have found that the best region of temperature is between 4 and $6^{\circ} \mathrm{C}$, but this value must be kept directly on the cathode not in the cooling bath. Secondly, the lower current density assured a better hydride purity, in terms of hydrogen content even though this has caused a significant retardation of the process. Following this procedure, we have obtained a very good reproducibility of the sample quality both in terms of hydrogen content and fcc structure purity. The hydrogen content, as measured by masspectroscopic method, has been always close to one in atomic ratio and X-ray analysis have shown the fcc hydride peaks only. We used the energy dispersive method of taking diffraction patterns. The interplanar spacings $d_{h k l}$ were calculated according to the equation:

$$
\mathrm{E}_{\mathrm{KeV}} * \mathrm{~d}_{\mathrm{hkl}} * \sin \theta=6.2 \AA * \mathrm{KeV}
$$

where E corresponds to peak position and $\theta$ is the fixed diffraction angle.

b.) High pressure apparatus: The high pressure apparatus used for establishing equilibrium hydrogen pressure above solid phase of fcc chromium hydride is presented in Fig. 1

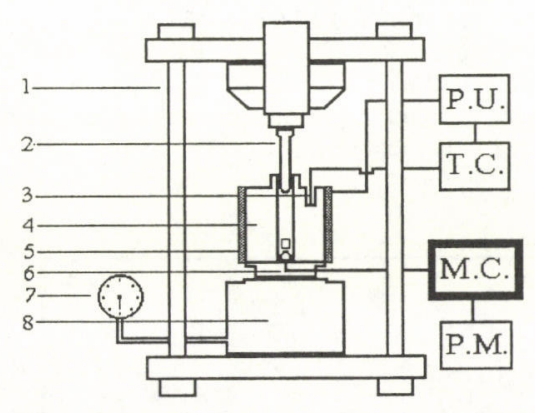

Fig.1. High pressure apparatus for the studies of metalhydrogen systems.(1 - ram, 2 - piston, 3 - temperature sensor, 4 - high pressure chamber, 5 - heater, 6 - high pressure capillary, 7 - dial gauge, 8 - hydraulic pump, see the text)

Basically, the apparatus consists of the high pressure unit, already used for the determination of the $\mathrm{p}-\mathrm{c}$ 
isotherms in metal-hydrogen systems[13] with modification introduced in another design by Baranowski and Bojarski [8], which allows for pressure measurements in room temperature, while high pressure chamber is kept at high temperature. The manganine resistance gauge was kept in high pressure cell [M.C.] filled with silicon oil to avoid the direct contact with hydrogen gas.

Two methods for the determination of equilibrium hydrogen pressure of chromium hydride have been employed. The first: hydride sample has been placed in the high pressure apparatus with hydrogen at pressure of about $0.2-0.3 \mathrm{GPa}$ at room temperature. Next, the high pressure chamber was heated to $150^{\circ} \mathrm{C}$, the same temperature as for the hcp hydride measured before[8], and the sample has been kept inside the chamber during two days, which are necessary to reach the equilibrium between gaseous hydrogen and the hydride phase. The temperature stability of about $0.5^{\circ} \mathrm{C}$ was assured by temperature controller [T.C.], power unit [P.U.] and platinum resistance sensor (3). Sample of the hydride, after such a treatment, has been taken out and analyzed by masspectroscopic and X-ray methods.

The second: The increase of pressure due to hydride decomposition has been monitored up to the stationary value as used before[14]. Knowing the geometry of the chamber and the equation of state of hydrogen, the volume of hydrogen, released during the decomposition process, has been calculated and then in a few steps pressure-composition relationship has been determined.

\section{Result and discussion}

\section{Equilibrium pressure determination.}

In the first method, a large range of discrepancies of pressure has been observed, nevertheless, a fit to the data shows that the pressure of hydrogen above fcc $\mathrm{CrH}$ is smaller than that for hcp obtained from Baranowski and Bojarski work [8]. The p-c isotherm for the decomposition of the fcc chromium hydride phase, obtained by second method, along with the data for the hcp hydride [8] is presented in Fig. 2

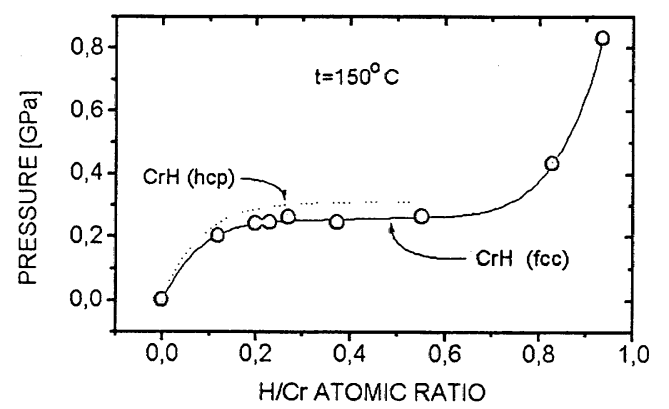

Fig. 2. Decomposition isotherms for the chromium hydrogen system at $150^{\circ} \mathrm{C}$.
The equilibrium pressure as determined from the two methods has been established as: $p=0.25 \pm 0.005 \mathrm{GPa}$ and is lower than for hcp phase by about $0.05 \mathrm{GPa}$. This value has been used for calculating standard free energy of formation of the fcc chromium hydride, following previously employed procedure for the hcp hydride [8]. For the stoichiometric chromium hydride the chemical potential at equilibrium conditions can be written as:

$$
\mu_{C r H(p, T)}=\mu_{C r(p, T)}+1 / 2 \mu_{H_{2}(p, T)}
$$

where $\mu_{\mathrm{Cr}(\mathrm{p}, \mathrm{t})}$ and $\mu_{\mathrm{H} 2(\mathrm{p}, \mathrm{t})}$ denote the chemical potentials of chromium and gaseous hydrogen respectively, both components being in equilibrium with the solid chromium phase. In fact, the solid solution of hydrogen in chromium ( $\alpha$-phase) should be considered instead of pure chromium, but this is usually neglected as the contribution to chemical potential from the $\alpha$-phase is small. The chemical potential of chromium hydride at temperature $150^{\circ} \mathrm{C}$ and equilibrium pressure $2500 \pm 50$ bars was calculated as equal to $2450 \pm 50 \mathrm{cal} / \mathrm{mole} \mathrm{H}_{2}$. In order to reduce this value to the standard conditions i.e. $25^{\circ} \mathrm{C}$ and 1 bar following formula has been used:

$$
\Delta G^{0}=\mu_{C r H(p, t)}+\int_{p}^{1} V_{C r H}-\int_{150}^{25} S_{C r H} d T
$$

where $\mathrm{V}_{\mathrm{CrH}}$ and $\mathrm{S}_{\mathrm{CrH}}$ denote the molar volume and entropy of chromium hydride, we have obtained the standard free energy of formation of the fcc chromium hydride equal to $\Delta \mathrm{G}^{0}=3960 \pm 50 \mathrm{cal} / \mathrm{mole} \mathrm{H}_{2}$

This value is about $200 \mathrm{cal} / \mathrm{mole} \mathrm{H}_{2}$ lower than the value for the hcp chromium hydride. By repeating these calculations in the opposite direction, the equilibrium pressure above fcc chromium hydride at $25^{\circ} \mathrm{C}$ has been determined as $\mathrm{p}_{\mathrm{eq}\left(25^{\circ} \mathrm{C}\right)}=0.056 \pm 0.005 \mathrm{GPa}$.

Lower value of the equilibrium pressure at room temperature may suggest also a lower pressure of formation of the fcc chromium hydride as compared to the hcp phase, but the attempt to form fcc hydride phase from the elements in diamond anvil cell up to 10 $\mathrm{GPa}$ at $25^{\circ} \mathrm{C}$ has been unsuccessful, using the method described before[15]. The most probable reason for this failure, we believe, was that the temperature was too low for the lattice transformation from the bcc chromium metal to the fcc of hydride phase. If we assume, according to the general observation regarding the transition metal hydrides, that the entropy of formation of this group of hydrides is almost the same and equal to: $25 \mathrm{cal} / \mathrm{mole} \mathrm{H}_{2}$, then the enthalpy of formation of the fcc chromium hydride could be evaluated. Under above stated assumption, the enthalpy of formation of fcc chromium hydride has been determined as $\Delta H^{0}=-3490 \pm 50 \mathrm{cal} / \mathrm{moleH}_{2}$. This value is of about 200 calories more exothermic than the 
value of the hcp hydride as determined elsewhere $[10,11]$.

2. The equation of state

The chromium-hydrogen system presents unique feature among other metal-hydrogen systems as the two hydride phases coexist in the same thermodynamic conditions. As it was shown above, thermodynamic functions of formation of both phases are also similar. Since the molar volume of the fcc phase is smaller than the value of the hcp, one could expect a transformation from the fcc to the hcp structure under high pressure. Such a transition has been observed for the manganese hydrogen system[16]. In order to follow this idea we have determined the equation of state for the fcc type of hydride using diamond anvil cell technique up to 37 $\mathrm{GPa}$ by energy dispersive X-ray diffraction method, as described elsewhere[17]. The results are presented in Fig.3 along with data of pure chromium and hcp chromium hydride.

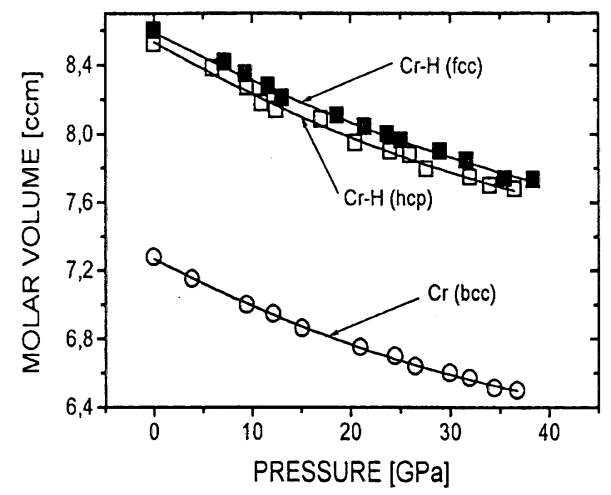

Fig. 3. Molar volumes as a function of pressure for the chromium metal, hcp and fcc chromium hydrides.

As it can be seen in Figure 3, no indication of the phase transition in the pressure region of investigation has been found. There are two probable reasons for the absence of phase transition. Firstly: due to relatively small difference between volumes of both the fcc and the hcp phases, the pressure applied was too low to induce phase transformation. Secondly: the temperature of measurements, in our opinion, should be substantially higher than $25^{\circ} \mathrm{C}$, to accelerate the mobility of chromium atoms in the hydride lattice. Solid lines in Fig. 3 represent fits of the data to the Birch-Murnaghan equation of state giving the values of $\mathrm{B}_{0}$ and $\mathrm{B}_{0}:(245 \pm 7,5,5),(240 \pm 7),(238 \pm 5)$ for the bcc chromium, hcp $\mathrm{CrH}$ and fcc $\mathrm{CrH}$ respectively. As it can be also noticed from data presented in Figure 3, that the introduction of a hydrogen atom into the chromium lattice resulted in almost the same molar volume increase of both chromium hydrides. This means that the fcc chromium hydrides follow also general rule, first time observed by Baranowski et al. [18] for nickel and palladium hydrides (including their alloys) that the hydrogen particle volume in the hydrides lattice calculated as the difference between the volume of the host lattice with one hydrogen particle and the initial volume of the hydrogenless metal has the constant value equal to $2,8 \pm 0.2 \AA^{3}$. For the fcc chromium hydride, the so called, universal volume of hydrogen particle was calculated as equal to: $2,6 \AA^{3}$ per hydrogen particle.

Regarding the compressibility of the fcc hydride as compared to the hcp phase and pure bcc chromium metal, it can be concluded that the volume of hydrogen species in metal lattice is pressure independent as stated first time by Fukai et al.[19] and confirmed later, for other transition metal hydrides, by Baranowski et al. [20].

\section{References}

[1] C.A. Snavely, Trans. Electrochem. Soc., 92, 537 (1947)

[2] C.A. Snavely, D.A. Vaugham, J.Am. Chem.Soc.,71 313 (1949)

[3] R.J. Roy, T.R.P. Gibb Jr. J.Inorg. Nucl: Chem., 29 341 (1967)

[4] A.D. Stock, K.J. Hardcastle, J. Inorg. Nucl. Chem., 321183 (1970)

[5] A. Knödler, Metaloberfl., 17331 (1963)

[6] B. Baranowski , K. Bojarski , Roczn. Chem., 45499 (1971)

[7] B. Baranowski, K. Bojarski, Roczn. Chem., 46525 (1972)

[8] B. Baranowski, K. Bojarski, Roczn. Chem. 46 1403 (1972)

[9] K. Wolf, Z. Physik. Chem.,(Leipzig) 246403 (1971)

[10] A. Sieverts, A. Gotta, Z. anorg.Chem. 1721 (1928)

[11] K. Bojarski, Thesis Inst.Phys. Chem. Pol. Acad. Sci. 1973. (in Polish)

[12] E.G. Ponyatowskij ,I.T. Belash, Dokl.Acad.Nauk. SSSR 2291171 (1976)

[13] B. Baranowski , M. Tkacz and W. Bujnowski, High Temp. - High Pressure $8 \quad 656$ (1976)

[14] B. Baranowski , M. Tkacz, Z. Phys. Chem. (N.F.) 13527 (1983)

[15] M.Tkacz, Polish J. Chem., 691205 (1995)

[16] S.M. Filipek, H. Sugiura and A.B. Sawaoka, High Pressure Res., 4354 (1990)

[17] M.Tkacz , B.Baranowski , Polish J.Chem., 66 1301 (1992)

[18] B. Baranowski, S. Majchrzak and T.B. Flanagan, J. Phys., F1 258 (1971)

[19] A. Fukizawa,Y. Fukai, J. Phys. Soc. Japan, 52, 2102 (1983)

[20] B. Baranowski, S. Majchrzak ,M. Tkacz, Pressure Dependence of Hydrogen Volume in Some Metallic Hydrides, in Molecular Systems Under Pressure, R.Pucci and G.Piccitto (eds), Elsevier Science Publ. 1991, p.p 139-145 